\title{
Recent epidemics and their impact on the society: COVID-19, SARS, MERS, H1N1
}

\author{
Hanzhi Zhang, a, * \\ ${ }^{1}$ Dunn School, Los Olivos, CA 93441, United States
}

\begin{abstract}
Even in 2021, people still have questions and concerns about the new Covid-19. It is very important for the public to learn about the disease and to understand how to protect themselves from it, which is a part of the public health's responsibility. Quarantine, social distance, and more facts about the disease will be beneficial for developing people's self-awareness when involved in a pandemic. Since Covid-19 is an airborne disease, people will have to keep their distance from each other and use masks if possible. Since lock-down passed down in China last year, the death rate and confirmed cases of covid-19 have been decreasing. While people want to go back to work and school, new methods have been come out to help people succeed during this pandemic. In order to help people prevent and protect themselves from Covid-19, this paper will systematically introduce the essential knowledge about Covid-19.
\end{abstract}

\section{Introduction}

Covid-19 is a newly discovered coronavirus that appeared in December, 2019. It is an airborne epidemic disease (a virus). This disease attacks people's immune and respiratory systems, leading to respiratory failure and multiple organs shutdowns. Once the virus enters the human body, it will start making its way to the immune system, causing early stage symptoms of the Covid-19. The early stage symptoms of Covid-19 are common symptoms that also occur in flu and cold. These symptoms are fever, cough, and shortness of breath. In addition, there are more symptoms that vary from one another. It is hard to tell because they share similar symptoms with common non-lethal illnesses. That is another reason why it spread so fast in the beginning of this Covid-19 pandemic period. The first case of Covid-19 was reported in Wuhan, China on December 31, 2019. In less than a month, the disease spread like wildfire around the world. Day by day, the confirmed case and mortality went up higher and higher. In the third month after the first incident, the World Health Organization officially announced the news about Covid19. Soon after, government officials made their announcement and started planning their way to fight the disease. That is also when quarantine, social distance, and masks started to come out. Covid-19 has limited people's freedom and changed their life with its sudden appearance. Everything seems to be going downhill. With the increasing case of numbers, hospitals are overloaded with patients, and medical resources are running out since most medical industries were forced to shut down. Multiple learning hospitals or academic hospitals are also taking overloading work [1]. Homeless people with no financial support had to try to survive on their own, and bodies are left in shadowy corners and under the bridges. Students no longer go to school, and parents can't even go to work. When everything is put on a graph, the only increasing line we'll see is the mortality rate and confirmed cases. Everything else is going the opposite. In the fall of 2020, part of the school and companies had resumed their sessions with the new policy that came along with it. During school and work, people are told to practice social distancing. Moreover, it is a requirement to wear a mask when someone is nearby. Every once a while, people will need to take the PCR test to confirm that they are not Covid-19 positive so they don't have the risk of spreading the disease. To confirm the patient's result for Covid-19, they will have to participate in a PCR test. PCR, commonly used in biology, stands for polymerase chain reaction, which is also be called molecular photocopying. It is used to "amplify" and make copies of small fragments of the DNA. First, the sample will be heated to denature the DNA so it can be separated into two single-stranded parts followed by Taq polymerase. Therefore, it can be synthesized into two new strands of DNA.

Each new strain contains one old strand and one new strand, and each copy can be used to create two new copies, and so on. The replication can be repeated 30 to 40 times, resulting in billions of copies of the original DNA fragments. The whole PCR cycle is automated and can be completed in a few hours. It is controlled by a machine called a thermal circulator, which is programmed to change the temperature of the reaction every few minutes to denature and synthesize the DNA [2]. At present, there is no cure for this kind of disease, but there are many drugs currently in clinical trials. Not all of the patients with Covid-19 need medical treatment. Because some of them have a strong immune system so they are more likely to recover on their own without having any kind of medical

Corresponding author:a reiner_zhang@student.dunnschool.org 
support. Currently, hospitals have been giving supplemental oxygen and advanced organ support for patients with respiratory failure, septic shock, and multiorgan failure. With all the treatments that are currently going through clinical trials, we are hoping to see more effective treatments for patients to use as more and more studies come out [3].

\section{The Impact of COVID-19}

Over the last year, people have been trying to come out with plans to help them resume their normal life in many ways. The first thing on people's minds is their job. There were a significant number of people who lost their job due to covid, and people are trying to find their ways to address this current situation that has been limiting their freedom for a very long time. Some companies started to reorganize their workspace in order to prevent the spread of covid. Therefore, protective screens were places and new policy came. People who come in for work will have to wear masks and clean up the working area with clean wipes and hand sanitizer. Some of them are even forced to wear gloves due to their jobs such as doctors and nurses who need to be in close contact with their patience. Many schools also considered a similar plan while other schools decided to do remote learning through zoom and other online platforms for schooling. But the important point is that Covid-19 is not going to hurt people's physical health, but also our mental health. It's just like being in prison. People now have to stay at home most of the time. Some that live by themselves have no other kinds of human interaction with others, which can create mental stress due to isolation. Many students who returned from online school have problems communicating with others and some even have social anxieties. [4] There has also been a great increase of requirements for counselors and therapists. People's mental health suffered badly from the isolation.

\section{Discussion}

Before the appearance of Covid-19, there were also several other epidemics such as SARS, MERS, and the Spanish Flu (H1N1). SARS and MERS are also coronaviruses that belongs in the same group with Covid19; [5] H1N1 is a flu that started in the middle of World War One that infected more than one-third of the human population. All of them had a great impact on human and human history. Because when an epidemic occurs, most of the time people will have to go into isolation. The community and its people will all be affected. Due to isolation or quarantine, people can't make any kinds of physical contact with each other their freedom are limited. Some can't go to work or school, and society seems like coming to a stopping point or a slower version compared to its normal routines.

\subsection{SARS}

SARS stands for the severe acute respiratory syndrome. It is a viral respiratory disease caused by a coronavirus. It's also called the SARS-associated coronavirus (SARSCoV).[5] SARS' first outbreak started in Beijing, China in February 2003.[6] Once the public health apartment officially announced SARS to the public, the Chinese government also passed out a lockdown. People had to stay home and stay isolated from others. Within a few days, the sickness spreads like a wildfire. There were more than twenty countries that had confirmed cases of SARS in their country, and about more than eight thousand people died due to SARS. Some patients who recovered from SARS also had lifelong after effects such as pulmonary fibrosis. In the end, the virus could survive in high temperatures so it couldn't spread normally in the outdoor area, and it was more than thirty celsius during the summer in Beijing, China. So during the summer of 2003, the disease faded away due to the outdoor temperature. Covid19 is very similar compared to SARS. For example, they are both airborne diseases and they have pretty much the same symptoms. Both of them are classified as a part of the coronavirus group, but they are different individual diseases when you compare them. The early stage symptoms of SARS are fever, chills, rigors, headache, malaise, and muscle pain. Moreover, there are mild respiratory symptoms, dry, non-productive cough or dyspnoea, and hypoxemia. Some Of them may also have diarrhea. [7] SARS attacks people's respiratory and immune systems and it can lead to multiple organ failure and death. Hospitals were overloaded when more and more people got sick. People who couldn't afford to pay for medical care got thrown out of the hospital. There is also no treatment or cure for SARS. People who survive from SARS usually have a strong immune system, therefore young children and older people have higher chances of dying compared to others.

\subsection{MERS}

The second disease is MERS. MERS stands for Middle East Respiratory Syndrome which is one of the coronaviruses (MERS-CoV). [5] Another name for MERS is Camel flu. MERS is just like other coronaviruses, it attacks people's respiratory systems. Usually, patients with MERS start with fever, cough and shortness of breath. The first officially reported case of MERS was in Saudi Arabia in September 2012. After a thorough investigation, they found out the first case occurred in Jordan in April 2012. Since 2012, about twenty-seven countries have reported their countries' cases of MERS. With a mortality rate of 35 percent, there're about 851 people who died from MERS, and more from other different complications during that time.[8] When MERS was spreading in Jordan and Saudi Arabia, the government didn't take any actions to address the situation. But the record of death for MERS was much lower compared to Covid-19 and SARS. MERS' cases slowly decreased and became stable in the countries that have MERS cases. Currently, there is no cure for MERS. But it can be treated with some medical care. The patients will require to rest. Usually, the doctors will give out fluids, pain relievers, and oxygen therapy for the patient. Since coronavirus mainly attacks people's immune systems, it is important for people to stay rested and hydrated. At the 
same time, nutrients and vitamins will also need to be taken into consideration. MERS originally started in 2012, and it still exists in nowadays. But right now, it is no longer spreading across multiple countries. [9]

\subsection{H1N1}

H1N1 is a flu virus that started in the United States in 2009 during World War One. It is classified as an influenza A virus that's similar to normal seasonal flu. [10] It is also one of the flu virus strains that can cause the seasonal flu, and they also have the same symptoms. Another name for $\mathrm{H} 1 \mathrm{~N} 1$ is the swine flu. Because H1N1 is very similar to influenza viruses for pigs in North America. H1N1 is combined with viruses that come from pig, bird and human. It is the first global flu pandemic in forty years. The symptoms of H1N1 are fever, cough, sore throat, runny or stuffy nose, body aches, headache, chills and fatigue. There are about 60.8 million cases during the pandemic. About 12,469 people died in the United States and between 151,700 to 575,400 people died due to the H1N1 flu virus. The majority of people that were affected by this illness are children and middle-aged adults. There is no cure for H1N1. But in November of 2009, researchers developed vaccines for $\mathrm{H} 1 \mathrm{~N} 1$, and many people received it. But because the vaccine has an age limit for people, so not everyone who has H1N1 can get it. Even though the vaccine can help people to encounter the flu, but there are still side effects of it as our immune system tries to learn this new virus. The side effects are usually wheezing, hypersensitivity reactions, and headaches. It usually las no longer than a few days, and there is a low percentage of people who are likely to have these side effects. [11]

\subsection{COVID-19}

Covid-19 is one of the coronaviruses that was newly discovered in December 2019. The symptoms of Covid19 are cough, shortness of breath/difficulty breathing, fever, chills, body/muscle aches, vomiting, diarrhea, and loss of taste/smell.[5] Covid-19 attacks people's immune systems first and causes the early stage symptoms of Covid-19. It will also attack the respiratory system and cause many other organs to go into organ failure. In order to maintain the patients' health and stable them. They will have supplemental oxygen and advanced organ support to help them [12]. There is currently a new vaccine just for Covid-19 and it is working for most of the people who's over the age of sixteen and eighteen. The doctors and researchers are still trying more effective and less harmful medicine for the patients. (going through clinical trials) In March, 2020, there were starting to have Covid-19 vaccine for people to take [13]. Vaccines are like tiny viruses people inject into their bodies. Where the human body's immune system will learn the disease and get used to it. So when the person gets exposed to the virus, the body will know how to react. It can also be described as "people who have the antibody". Such antibody is called immunoglobulin. It is a protein used by the immune system to identify and neutralize foreign substances such as bacterias and viruses.

\section{Conclusion}

There were many epidemics before the COVID-19, and many people died and suffered from these diseases. In 2021, COVID-19 is still black people away from resuming their normal life. In this case, in order to prevent the sickness from spreading more widely, it is important for people to understand that there are certain ways to help prevent and protect themselves from getting the disease or spread it to other people in the community. Even if the situation with COVID-19 is getting better with the new developed vaccine and treatment, it is still important for people to learn from this pandemic. If a new disease appears in the public just like COVID-19 again, the community and people can learn from the past and react faster to the new disease. Therefore, the cases and mortality rate can be the lowest. Everything will be recorded into human history as time passes. Moreover, people need to learn from history so that humanity can move on making fewer mistakes in the future.

\section{References}

1. Meschi, T., et al., Reorganization of a large academic hospital to face COVID-19 outbreak: The model of Parma, Emilia-Romagna region, Italy. European Journal of Clinical Investigation, 2020. 50(6): p. e13250.

2. Genome.gov. Polymerase Chain Reaction (PCR) Fact Sheet. 2020; www.genome.gov/about-genomics/factsheets/Polymerase-Chain-Reaction-Fact-Sheet].

3. Bobrovitz, N., et al., Lessons from a rapid systematic review of early SARS-CoV-2 serosurveys. medRxiv, 2020: p. 2020.05.10.20097451.

4. Kuhfeld, M., et al., Projecting the Potential Impact of COVID-19 School Closures on Academic Achievement. Educational Researcher, 2020. 49(8): p. 549-565.

5. Guarner, J., Three Emerging Coronaviruses in Two Decades. Am J Clin Pathol, 2020. 153(4): p. 420-421.

6. Organization, W.H. Severe Acute Respiratory Syndrome (SARS). www.who.int/healthtopics/severe-acute-respiratorysyndrome\#tab=tab_3].

7. UPMC. COVID-19 Symptoms, Diagnosis, \& Treatment. https://www.upmc.com/coronavirus/covid-19].

8. Organization, W.H. Middle East Respiratory Syndrome Coronavirus (MERS-CoV). www.who.int/news-room/fact-sheets/detail/middleeast-respiratory-syndrome-coronavirus-(mers-cov)].

9. Prevention, C.f.D.C.a. Middle East Respiratory Syndrome (MERS). www.cdc.gov/coronavirus/mers/index.html].

10. Prevention, C.f.D.C.a. Pandemic Influenza. 2020; www.cdc.gov/flu/pandemic-resources/index.htm]. 
11. Prevention, C.f.D.C.a. Vaccine against 2009 H1N1 Influenza

Virus. www.cdc.gov/h1n1flu/vaccination/public/vaccinatio n_qa_pub.htm].

12. Prevention, C.f.D.C.a. Clinical Questions about COVID-19: Questions and Answers. 2021; www.cdc.gov/coronavirus/2019-ncov/hcp/faq.html].

13. Prevention, C.f.D.C.a. CDC COVID Data Tracker. covid.cdc.gov/covid-data-tracker/\#datatracker-home]. 